\title{
Discrimination and representation of relative numerosity in a bisection task by pigeons
}

\author{
LaVinia TAN ANd Randolph C. GRaCe \\ University of Canterbury, Christchurch, New Zealand
}

\begin{abstract}
We trained 4 pigeons in a numerical bisection task to discriminate between pairs of keylight flashes with a ratio of 1:3 (2 vs. 6,4 vs. 12 , and 8 vs. 24) that were presented in a sample phase. Responses to the blue key were reinforced after a sequence of a larger number of flashes, and responses to the white key were reinforced after a sequence of a smaller number of flashes. The intervals between flashes in the sample phase were randomized to attenuate the covariation of temporal cues with flash number. Pigeons responded accurately in each of the discriminations, with typically $85 \%-90 \%$ correct responses. Transfer tests showed that the proportion of large responses increased with number and performance generalized to larger values outside the training ranges. Psychometric functions superposed when plotted on a relative scale, and estimates of Weber fractions were approximately constant, suggesting that variability was scalar. However, contrary to previous research in nonhumans, bisection points were located at the arithmetic, not geometric, mean. Hierarchical logistic regressions confirmed significant control over responding by number beyond that attributable to temporal cues. These results show that pigeons are able to respond accurately in a relative numerosity discrimination with successively presented visual stimuli, although the nature of the numerical representation and response rule remains unclear.
\end{abstract}

Relative numerosity discriminations have played a major part in the investigation of nonverbal numerical abilities and the representation of number by both humans and nonhuman animals. These discriminations are considered to be among the most basic numerical discriminations, involving fairly imprecise, dichotomous judgments of few versus many (Davis \& Pérusse, 1988). The capacity for relative numerosity discrimination does not necessarily require a true concept of number, but it may serve as a basis for the development of more sophisticated numerical abilities, such as absolute number discriminations.

Previous studies examining the ability of nonhuman animals to spontaneously discriminate relative number have required subjects to make one response, selecting either the smaller or the larger item (e.g., Beran, 2001, 2004, 2007; Hanus \& Call, 2007; Hauser, Carey, \& Hauser, 2000; Olthof, Iden, \& Roberts, 1997; Rumbaugh, SavageRumbaugh, \& Hegel, 1987; vanMarle, Aw, McCrink, \& Santos, 2006). However, these studies have often been limited by covariation between number and nonnumerical cues, such as number and volume or surface areaparticularly since the tasks often involve food as stimuli. A stronger test of relative numerosity - and a more sophisticated understanding of number - would require subjects to categorize exemplars as small or large. This type of discrimination is tested in numerical bisection tasks. A typical bisection procedure involves baseline training with two exemplars or samples differing in number while controlling for other cues such as area or stimulus duration, followed by testing with novel intermediate values. Subjects are required to produce one response following the small sample and to produce another response following the large sample.

Research has studied how nonhumans bisect both visual and auditory numerical stimuli (e.g., Alsop \& Honig, 1991; Fernandes \& Church, 1982; Honig \& Stewart, 1989; Meck \& Church, 1983; Roberts \& Mitchell, 1994), as well as response number (Emmerton, 1998; Emmerton \& Renner, 2006; Fetterman, 1993; Rilling \& McDiarmid, 1965). These have found largely positive results, showing that animals are able to respond differentially to stimuli that differ in relative numerosity, successfully categorizing them as large or small, and are able to transfer this performance to novel numerical values both within and outside the training range.

Bisection procedures also provide a means of investigating numerical representation through the analysis of response characteristics - in particular, response variability. Examination of psychometric functions (proportion of the large key choices as a function of number) provide information about how responding may be determined.

There are three forms of subjective numerical scales that have been proposed to support numerical discriminations. One is a precise representation of number, which is limited to human verbal counting processes; the other two are approximate and are found in human and nonhuman nonverbal discriminations of number. The precise repre- 
sentation of number consists of a linearly spaced number scale with constant generalization or variability between values and allows exact 1:1 mapping between objective and subjective number. It is the typical scale structure that would be used by an adult human who possesses a true concept of number and is proficient in numerical discriminations. The approximate representations are a logarithmic numerical scale with constant generalization between values and a linear numerical scale with increasing generalization between values.

Both types of approximate representation possess a common feature: As numerical magnitude increases, the representations of individual numbers become increasingly more variable due to compressed scaling in the logarithmic scale and increasing generalization between numbers in the linear scale. Consequently, both types predict scalar variability, where response variability increases proportionally to mean numerical magnitude, such that relative response variability remains constant as number increases. This contrasts with the binomial variability obtained in humans in verbal counting procedures, where relative response variability decreases as number increases (e.g., Brannon, 2006; Cordes, Gelman, Gallistel, \& Whalen, 2001).

Psychometric functions, obtained by plotting the proportion of large responses as a function of sample number, are generally ogival in form. Generally, little to no large responses are produced in response to the presentation of small numbers located below or close to the small sample number used in baseline training, but they do increase as sample number increases, reaching a maximum located around the large sample number value experienced in baseline. One robust finding is the superposition of psychometric bisection functions when plotted on a relative scale (e.g., Fetterman, 1993; Meck \& Church, 1983; Roberts, 2005). That is, when normalized, functions overlap, regardless of the absolute numerical range with which they were originally obtained. Superposition is consistent with both Weber's law and scalar variability.

The bisection point (BP), also known as the indifference point or point of subjective equality (PSE), can be calculated from the psychometric function. The BP is the numerical value for which the proportion of large responses is at chance $(50 \%)$. Their location may provide some information about the nature of the underlying numerical scale (Gibbon, 1981). If representations of number are scaled logarithmically, with constant generalization between values, and discriminations are based on the relative similarity of ratios of the current stimulus to each training stimulus (i.e., a similarity judgment rule), the BP should be located at the geometric mean. This is also predicted if the scaling of the numerical representation is linear with scalar response variability and the same rule is applied. A different BP with a linear number scale exhibiting scalar variability is predicted if a proximity rather than similarity rule is used; assuming that subjects respond on the basis of which anchor value most likely generated the noisy test stimulus, the BP should occur at the harmonic mean. Finally, if numerical representations are linearly spaced, with constant variability, then BPs should occur at the arithmetic mean (Gibbon, 1981).
Research has found mixed results with respect to the location of BPs. The majority of counting and timing experiments in nonhumans have obtained BPs at the geometric mean (e.g., Fetterman, 1993; Roberts, 2005), although others have obtained BPs at the harmonic mean (e.g., Fetterman, Dreyfus, \& Stubbs, 1985) and others between the geometric and harmonic means (Fetterman, Stubbs, \& Dreyfus, 1986). Additionally, some research in humans has found BPs at the arithmetic means (Droit-Volet, Clément, \& Fayol, 2003). Overall, previous bisection data do not provide a clear indication of the underlying representation used by nonhumans in numerical discrimination tasks.

However, most research using numerical bisection procedures has involved stimuli in which temporal and numerical characteristics have been confounded. Previous research has shown that the relative control of responding by these variables is influenced by training (Roberts \& Mitchell, 1994). Although many studies have demonstrated control by number through the manipulation of temporal and numerical variables in transfer tests, or via statistical analyses, the covariation between time and number during training may have reduced numerical control over responding.

The goal of the present study was to examine the performance of pigeons in a numerical bisection task in which the covariation of numerical and temporal cues was attenuated. The procedure was based on the numerical reproduction task developed by Tan, Grace, Holland, and McLean (2007), modified to investigate relative rather than absolute numerosity discrimination. Pigeons were presented with a sequence of response-dependent keylight flashes, after which they were required to make one of two responses. The flash rate and the cumulative duration of the sample phase were both randomized to reduce covariation of temporal cues with number. Subjects were initially trained and tested with relatively small values: 2 and 6 . Then, subjects were trained to discriminate between 4 and 12 flashes and between 8 and 24 flashes before replicating the 2-versus- 6 discrimination. Using multiple ranges allowed for a better investigation of other aspects of performance that might distinguish between logarithmic and linear scales, such as superposition of bisection functions and coefficients of variation. In addition, transfer tests included values two units outside of the upper and lower limits of the training range. Whereas previous experiments have generally examined transfer to numbers within the baseline values (with the exception of Emmerton \& Renner, 2006), the use of transfer test values outside of the training range yields a stronger test of numerical competence.

\section{METHOD}

\footnotetext{
Subjects

Subjects were 4 pigeons (Columba livia) numbered 181-184. All had experimental histories involving choice procedures, but they had had no exposure to numerical or temporal discriminations. Prior to the present study, they had participated in an experiment using a concurrent-chains procedure, in which they chose between terminal links that signaled 10 - and 20-sec delays to food and which changed unpredictably across sessions (Christensen \& Grace, 2008). The pigeons began training in the present study immediately after the prior
} 
study was completed. Subjects were maintained at approximately $85 \%$ of their free-feeding weights by additional feeding, when necessary, after experimental sessions. Water and grit were freely available in their home cages.

\section{Apparatus}

Four chambers were used, each containing a row of three keys. The chambers measured $40 \times 40 \times 32 \mathrm{~cm}$, with the row of keys situated $21 \mathrm{~cm}$ above the floor. The center key was located $16 \mathrm{~cm}$ from each wall, and the other two keys were located $8 \mathrm{~cm}$ on either side of the center key. Each key required a force of approximately $0.15 \mathrm{~N}$ for a response to be registered. The panel also contained a houselight, located $8 \mathrm{~cm}$ above the center key, and a food hopper, located $13 \mathrm{~cm}$ below. During reinforcement, the houselight and response keys were dark, while the hopper was illuminated and raised to allow access to the wheat. Fans attached to each chamber provided ventilation and masking noise during experimental sessions. Sessions were controlled and recorded by a computer running MED-PC software, located in an adjacent room.

\section{Procedure}

Sessions were conducted at approximately the same time each day, 7 days a week. Sessions ended when 72 trials had been completed or after $120 \mathrm{~min}$ had elapsed, whichever came first. Each trial was preceded by a 12 -sec intertrial interval (ITI), during which the houselight and keylights were dark. Trials consisted of a sample phase and a discrimination phase. At the start of the sample phase, the houselight was illuminated. Then, after a variable delay, the center key was illuminated red until a single response to the center key extinguished it. This sequence (delay, illumination of the center key, response) will be referred to as a flash. Baseline training involved two trial types, large and small, with sample phases consisting of $N$ or $N \times 3$ flashes. Trial types were randomly determined, subject to the constraint that there were 3 trials each with $N$ or $N \times 3$ flashes in every block of 6 trials, resulting in 36 large and small trials each per session.

To degrade the correlation between flash number and temporal cues, sample phase stimulus delays were determined using a doublerandomization procedure. At the start of a trial, an expected sample phase duration was selected randomly without replacement from a list of durations (in seconds): 5, 6, 7, 8, 9, 10, 11, 12, 13, 14, and 15. The programmed average interflash interval (IFI) for any particular trial was calculated as the expected sample phase duration divided by flash number. Finally, the actual individual IFIs were determined by multiplying the average IFI by a delay sampled without replacement from a distribution of 12 delays with an average of 1 sec generated by an exponential progression (Fleshler \& Hoffman, 1962). This procedure was expected to decrease the relationship between number and both cumulative sample phase duration and average flash rate (the inverse of the average IFI).

After the last flash had been completed, the houselight and all keylights were darkened for a 2 -sec retention interval. Following the retention interval, the houselight and the left and right keys were illuminated to signal the start of the discrimination phase. If two flashes occurred during the sample phase, a response to the white key was the correct discrimination response, whereas, if six flashes occurred, the correct response was a blue key response. The flash number-response key color association was the same for all pigeons and conditions, but the locations of the blue and white keys were randomized on individual trials. Once a key had been pecked, the discrimination phase ended, and both keylights were darkened.

If subjects made the correct response during the discrimination phase, reinforcement was presented. During reinforcement, the keylights and houselight were extinguished while the hopper was raised and illuminated for $4.5 \mathrm{sec}$. Incorrect responses were followed by a 5 -sec blackout and a correction trial. Correction trials were identical to the preceding regular trial, except that the correct keylight was illuminated at the beginning of the discrimination phase. A single peck to the illuminated key resulted in $1.5 \mathrm{sec}$ of hopper access.

Subjects received 127 or 128 sessions of baseline training with 2- and 6-flash trials until asymptotic performance was obtained for all 4 birds. Transfer tests were then conducted with the novel values $0,1,3,4,5,7$, and 8 . These new numbers were presented in probe trials, randomly arranged among the 2- and 6-flash baseline trials. In any given session, there were 3 trials each for four of the novel values and 2 trials each for three of the novel values, randomly determined, resulting in 54 baseline trials and 18 probe trials in each transfer test session. Thus, although the number of probe trials for each novel sample number may have differed slightly on any given session, the frequencies of the novel values were approximately equal across sessions. To reduce the likelihood that pigeons would discriminate probe and baseline trials, responses on probe trials were reinforced randomly $(p=.50)$. Analyses of probe trial performance comparing the first and second blocks of 5 sessions in the first and last 2-versus-6 baseline conditions found no significant effects or interactions, suggesting that random reinforcement on probe trials did not affect performance.

Pigeons then completed two conditions that required discrimination between 4 and 12 flashes and 8 and 24 flashes. Each condition consisted of at least 40 sessions of baseline training followed by at least 30 sessions of transfer testing. The decision to switch subjects between conditions was based on visual assessment of stability, given that at least these many sessions of baseline or transfer training had been completed. Transfer values were $0,2,6,8,10,14$, and 16 for the 4 -versus- 12 condition and $0,4,12,16,20,28$, and 32 for the 8 -versus- 24 condition. Pigeons 181 and 182 completed the 4 -versus- 12 condition first followed by the 8 -versus- 24 condition, whereas the order was reversed for Pigeons 183 and 184. After these conditions, all pigeons were returned to the original 2-versus-6 condition and completed 32 sessions of baseline training followed by 30 transfer sessions. Transfer sessions in the 4-versus-12 and 8 -versus- 24 conditions were identical - with the exception of sample numbers - to transfer sessions experienced in the 2-versus- 6 condition. The order of conditions and the number of sessions of training and transfer testing are shown in Table 1.

Data from the last 10 sessions of baseline training and baseline replication, as well as from the first 10 sessions of transfer testing, were aggregated and used for analysis. A significance level of $p<.05$ was used in all statistical tests.

\section{RESULTS}

\section{Baseline Performance}

The average percentage of correct responses for small and large trials is shown in Figure 1 for all four conditions.

Table 1

Order of Experimental Conditions and Number of Sessions in the Baseline Training and Transfer Test Sessions of Each Condition for Individual Subjects

\begin{tabular}{|c|c|c|c|c|c|c|c|c|c|}
\hline Subject & \multicolumn{3}{|c|}{ Condition 1} & \multicolumn{2}{|c|}{ Condition 2} & \multicolumn{2}{|c|}{ Condition 3} & \multicolumn{2}{|c|}{ Condition 4} \\
\hline 181,182 & \multicolumn{3}{|c|}{2 vs. 6} & \multicolumn{2}{|c|}{4 vs. 12} & \multicolumn{2}{|c|}{8 vs. 24} & \multicolumn{2}{|c|}{2 vs. 6} \\
\hline \multirow[t]{2}{*}{183,184} & & 2 vs. 6 & & $8 v$ & & $4 v$ & & & \\
\hline & Baseline & Transfer & Baseline & Baseline & Transfer & Baseline & Transfer & Baseline & Transfer \\
\hline No. sessions & $127 / 128$ & 49 & 72 & 42 & 31 & 60 & 42 & 32 & 33 \\
\hline
\end{tabular}




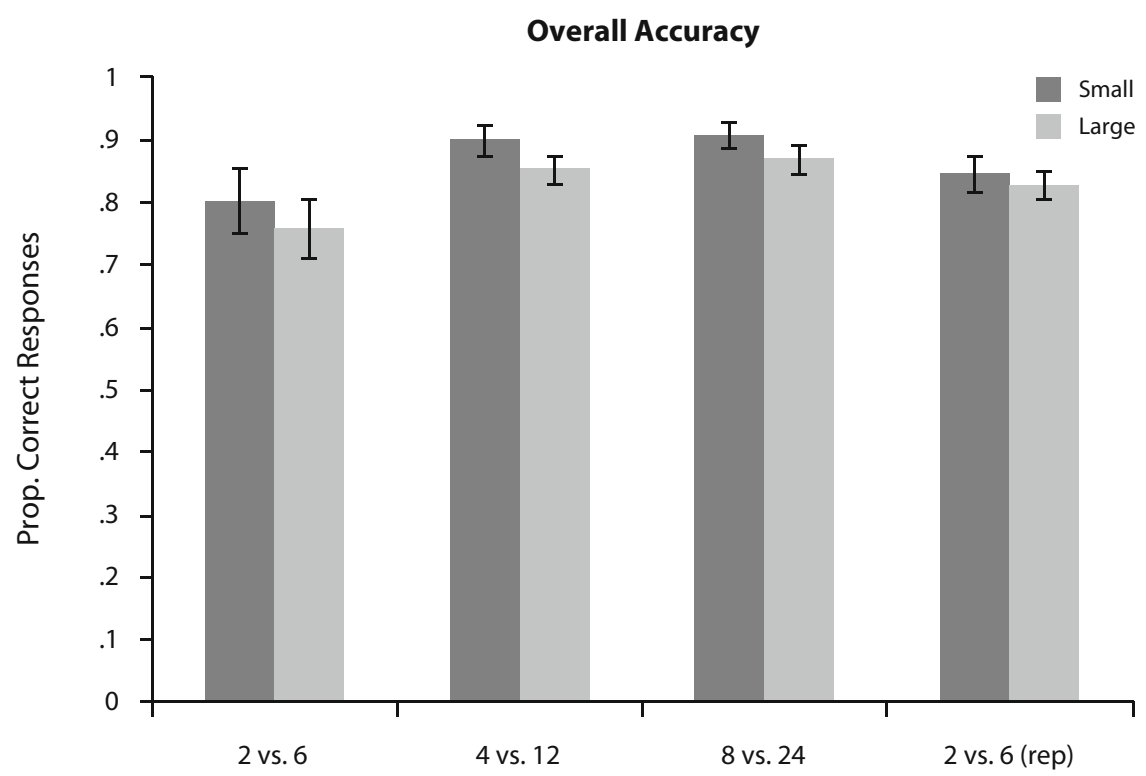

Figure 1. The average proportions of correct responses for large and small trials in each condition. Error bars indicate $\pm 1 S E$.

Pigeons performed at levels significantly above chance, averaging $78.1 \%, 87.7 \%, 88.9 \%$, and $83.4 \%$ correct in the 2-versus-6, 4-versus-12, 8-versus-24, and 2-versus-6 replication conditions, respectively. A repeated measures ANOVA with condition and trial type (small/large) found significant effects of condition $[F(3,9)=5.82]$ and trial type $[F(1,3)=49.89]$, but no interaction. Post hoc tests showed that accuracy in the first 2 -versus- 6 condition was lower than in the later conditions, which did not differ significantly. Consequently, the results reported below are based on the last three conditions. The effect of trial type indicated that accuracy was higher on small trials $(M=$ $88.4 \%)$ than on large trials $(M=85.1 \%)$. These results show that pigeons learned each of the three discrimina- tions successfully, with accuracy levels in the range of $85 \%-90 \%$ correct.

\section{Transfer Performance}

To provide a more detailed characterization of pigeons' numerosity discrimination, we calculated the probability of a large response during transfer sessions for each condition. The resulting psychometric functions are shown for the group means in Figure 2. Averaged data were representative of results for individual subjects. Positive transfer to novel values was demonstrated because the probability of a large response was directly related to the number of flashes and also extended to values outside of the training range $(0,1$ and 7,8$)$. Repeated measures ANOVAs

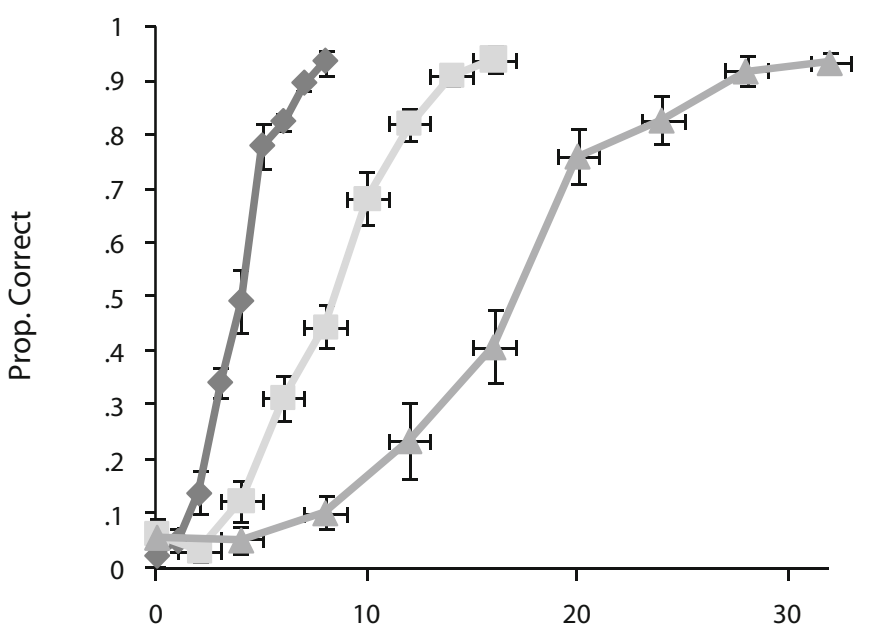

Flash Number

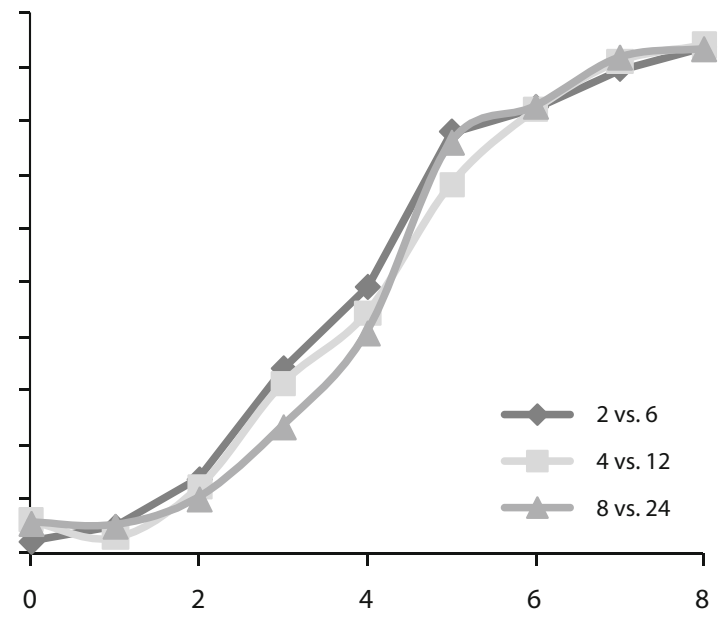

Flash Number/sec

Figure 2. Group mean psychometric functions plotted on absolute (left panel) and normalized (right panel) number scales. Error bars indicate $\pm 1 S E$. 
Table 2

Arithmetic Mean, Geometric Mean, and Bisection Points for All Subjects in All Conditions (Logistic Function Fit)

\begin{tabular}{|c|c|c|c|c|c|c|c|c|}
\hline \multirow[b]{2}{*}{ Condition } & \multicolumn{4}{|c|}{ Subject } & \multicolumn{2}{|c|}{$\underline{\text { Bisection Point }}$} & \multirow{2}{*}{$\begin{array}{c}\text { Arithmetic } \\
\text { Mean }\end{array}$} & \multirow{2}{*}{$\begin{array}{c}\text { Geometric } \\
\text { Mean }\end{array}$} \\
\hline & 181 & 182 & 183 & 184 & $M$ & $S E$ & & \\
\hline 2 vs. 6 & 3.87 & 4.21 & 3.84 & 3.83 & 3.94 & 0.091 & 4 & 3.46 \\
\hline 4 vs. 12 & 8.40 & 7.84 & 8.32 & 8.65 & 8.30 & 0.171 & 8 & 6.93 \\
\hline 8 vs. 24 & 15.90 & 18.60 & 15.71 & 16.77 & 16.74 & 0.661 & 16 & 13.86 \\
\hline
\end{tabular}

found a significant effect of flash number on the probability of a large response for the 2-versus- 6 discrimination $[F(8,24)=128.30]$, the 4 -versus-12 discrimination $[F(8,24)=118.85]$, and the 8 -versus-24 discrimination $[F(8,24)=78.85]$ (see Figure 2$)$.

Testing for transfer to values outside the training range, we conducted planned comparisons to compare the proportion of large responses to the two lowest transfer test values relative to the lower baseline training value and to the two highest transfer test values relative to the higher baseline training value. These comparisons found that the proportion of large responses in the 2-versus- 6 condition was significantly greater on 7- and 8-flash trials than on 6-flash trials $[F(1,3)=13.32]$, but there was no significant difference between the proportion of large responses on the 2-, 0 -, and 1-flash trials $[F(1,3)=6.13, p=.089]$. In the 4-versus- 12 condition, a planned comparison found no significant difference between the proportion of large responses for the two lowest transfer values, 0 and 2, and the lower baseline training value, $4[F(1,3)=5.43]$. The proportion of large responses for the two highest transfer values, 14 and 16, was significantly higher than the higher baseline training value, $12[F(1,3)=13.31]$. Responding in the 8 -versus- 24 condition was similar to that in the 4 -versus-12 condition. Planned comparisons found no significant difference between the lowest transfer test values, 0 and 4 , and the baseline value, $8[F(1,3)=3.68]$, and a significant difference between the highest transfer test values, 28 and 32, and the baseline value, $24[F(1,3)=$ 13.01]. The failure to find significant differences among the lower extreme values may be due to floor effects in responding: Often, the proportion of large responses to the smallest sample number (i.e., 2, 4, or 8 in the 2-vs.-6, 4-vs.-12, and 8-vs.-24 conditions, respectively) was less than 0.1 , restricting any further decrease in the proportion of large responses made. Overall, these results confirm that transfer successfully occurred for flash numbers outside the range included in training - at least for larger numerosities.

When the psychometric functions for the three different conditions were plotted along the same scale (by dividing the values for the 4 -vs.- 12 and 8 -vs.-24 conditions by 2 and 3 , respectively), the functions superposed, suggesting constant relative variability across the three different number scales. These can be seen in the right panel of Figure 2. A repeated measures ANOVA was conducted with the individual data, using condition and $N / S$ (sample number divided by the small anchor value) as predictors, and found a significant effect of $N / S[F(10,90)=334.261]$, but no significant effect of condition or interaction.
To provide estimates of the BPs for individual subjects, a two-parameter logistic function was calculated and fitted to the baseline and transfer test data:

$$
P(\text { large })=\frac{1}{1+e^{-A(n-B)}},
$$

where $n=$ number of flashes, $A=$ slope of the middle part of the function, and $B=$ the BP. These functions provided a good description of the data, accounting for between $97 \%-99 \%$ of variance for each subject in each condition.

The BP estimates were tested to determine whether they were located closer to the geometric mean or to the arithmetic mean of each scale. The predicted BP values, including the geometric and arithmetic means of the baseline training values in each condition, are shown in Table 2. All BPs were located closer to the arithmetic mean than to the geometric mean. Results of single-sample $t$ tests found no significant difference between BPs and the arithmetic means for the 2 -versus- 6 discrimination $[t(3)=$ $0.70]$, the 4 -versus-12 discrimination $[t(3)=1.78]$, and the 8 -versus-24 discrimination $[t(3)=1.12]$. However, BPs were found to be significantly different from the geometric means $[t(3)=5.22$, for the 2-vs.-6 discrimination; $t(3)=8.05$, for the 4-vs.-12 discrimination; and $t(3)=$ 4.37, for the 8-vs.-24 discrimination].

Difference limens (DLs) were also calculated for each subject and each condition by interpolating the numerical values at which the proportion of large responses that were made equaled $25 \%$ and $75 \%$ and halving the difference between these values. The DL provides a measure of response variability and represents an estimate of the smallest value required for a difference in numerosity to be discriminated. DLs are plotted in the upper panel of Figure 3. Two different patterns in DL values emerged: Pigeons 182 and 184 showed an increase in DLs as scales increased, whereas DL values for Pigeons 181 and 183 increased from the 2-versus-6 to the 4-versus-12 discrimination, but they did not increase for the 8 -versus- 24 condition. A repeated measures ANOVA obtained a significant effect of condition on DL values $[F(2,6)=8.89]$. For all subjects, the numerical difference necessary to achieve a difference in responding increased as the numbers of flashes increased; a trend analysis confirmed a significant linear trend $[F(1,3)=13.62]$. The increasing linear trend in DL values is consistent with scalar variability.

To assess changes in relative response variability as a function of flash number, we calculated Weber fractions by dividing the DL values by the BP, as in Emmerton and Renner (2006) and Fetterman (1993). The bottom panel of Figure 3 shows the resulting Weber fractions plotted 
A

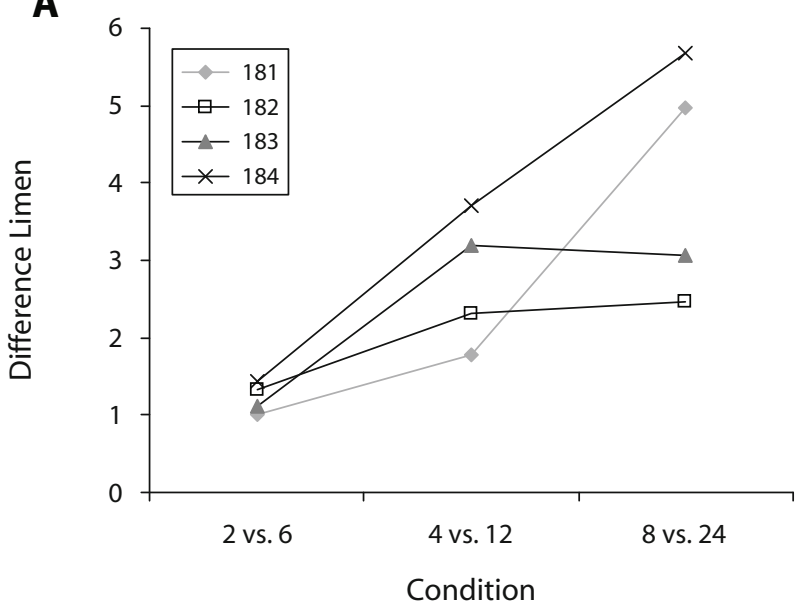

B

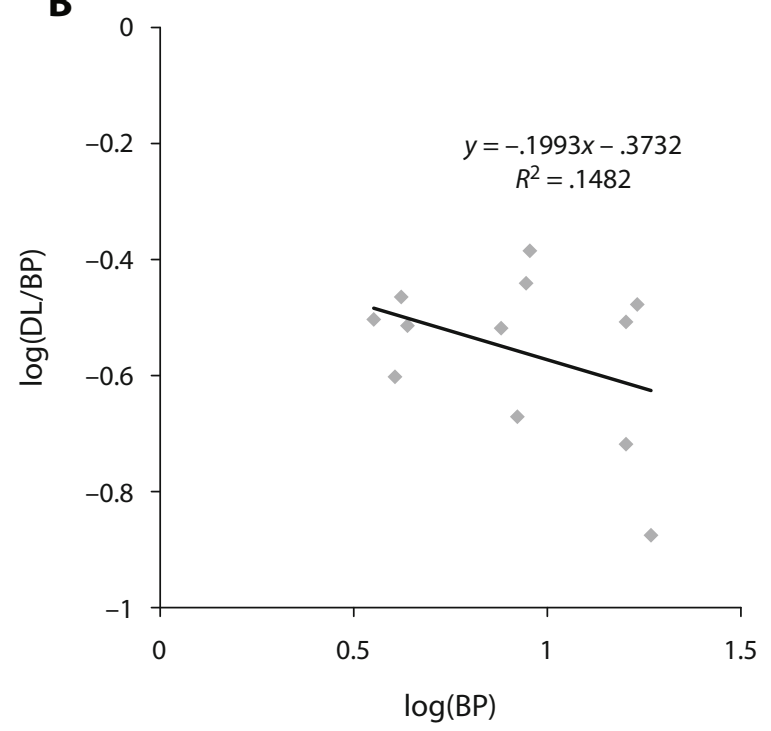

Figure 3. (A) Difference limen (DL) values for the 2-versus-6, 4-versus-12, and 8-versus-24 conditions. (B) Obtained Weber fractions (computed individually for each pigeon and condition) plotted on a log-log scale as a function of bisection point (BP).

against the BP values on a $\log -\log$ scale. Although the correlation between log Weber fractions and log BP was negative, suggesting that relative variability may have decreased, the relationship did not differ significantly from zero $\left(\beta=-0.38, R^{2}=.15\right)$. The lack of a systematic change in the Weber fractions also suggests that variability was scalar.

\section{Covariation of Temporal and Numerical Cues}

We conducted a series of analyses to determine the extent to which responding in the transfer test might have been controlled by temporal cues in addition to numerosity. The temporal variables computed for each trial were the duration of the sample phase and the flash rate (calculated as the flash number by the sample phase duration).

Mean cumulative stimulus durations and cumulative response delays for each condition are shown in the upper panel of Figure 4. Repeated measures ANOVAs found significant effects of number on sample phase duration in the 4-versus-12 condition $[F(8,24)=10.15]$ and the 8 -versus- 24 condition $[F(8,24)=8.77]$, but not in the 2 -versus- 6 condition $[F(8,24)=1.18]$. Additionally, significant linear trends were obtained in both the 4 -versus- $12[F(1,3)=33.18]$ and 8 -versus- $24[F(1,3)=$ 46.39] conditions. Flash rates were calculated for all trial types, except for 0-flash trials (see the lower panel of Figure 4). Similar to the baseline, flash rate increased more markedly with flash number, and the results of repeated measures ANOVAs showed significant effects of flash number in the 2 -versus- 6 condition $[F(8,24)=49.87]$, the 4-versus-12 condition $[F(8,24)=109.84]$, and the 8 -versus-24 condition $[F(8,24)=102.94]$. Significant linear trends were also found $[F(1,3)=485.67, F(1,3)=$ 174.98 , and $F(1,3)=537.59$ for the 2-vs.-6, 4-vs.-12, and 8-vs.-24 conditions, respectively]. Correlations between sample phase duration, flash rate, and flash number were positive and statistically significant in most cases. Averaged across subjects, the correlations with flash number for sample phase duration and flash rate were $.11(S E=$ $.01)$ and $.55(S E=.03)$ in the 2-versus-6 condition, $.21(S E=.01)$ and $.73(S E=.02)$ in the 4-versus-12 condition, and $.16(S E=.10)$ and $.75(S E=.02)$ in the 8 -versus-24 condition, respectively.

Because the procedure we used to randomize the IFIs did not completely eliminate the covariation between number and temporal cues, we conducted a series of hierarchical logistic regressions to test whether responding was controlled by flash number independently of temporal cues. These analyses used data from the first 10 sessions of transfer testing from each condition. In these analyses, sample phase duration and flash rate were entered at the first step, and flash number was entered at the second step. A summary is provided in Table 3. Flash number accounted for a significant amount of additional variance above and beyond the temporal variables - flash rate and sample phase duration - for all birds in all conditions, with the exception of 184 in the 8-versus-24 condition, which approached significance $(p<.08)$. In the full model, flash number was a significant (and the strongest) predictor of response for almost all subjects in all conditions. The exceptions were 184 in the 8 -versus-24 condition, in which the pigeon had greater control by both flash rate and sample phase duration, and 183 in the 4-versus- 12 condition, in which the pigeon had significant control by all three variables, of which sample phase duration was strongest. The amount of variance accounted for by flash number also approached significance for 181 in the 2 -versus- 6 condition. Control by sample phase duration was also significant for 181 and 182 in the 2-versus- 6 condition. The approximate variance accounted for (Nagelkerke $R^{2}$ ) by the full model averaged .60 across birds and conditions ( minimum $=.37$; maximum $=.80$ ). These results show that, in 11 of 12 cases (the exception being Pigeon 184 in the 8 -versus-24 condition), significant control $(p<.05)$ by flash number was obtained over responding beyond that attributable to flash rate and sample phase duration. 

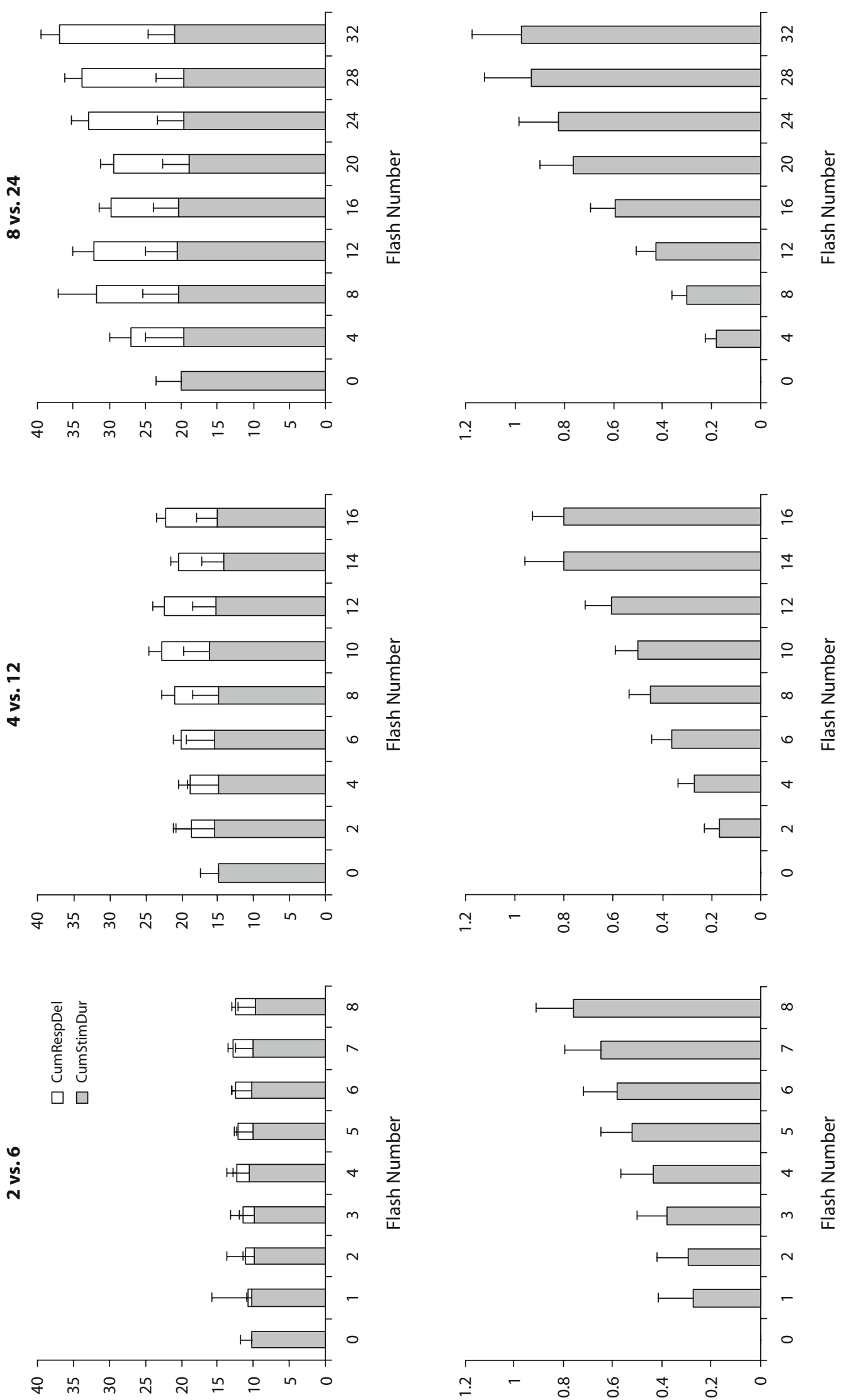

(गәS) uo!̣eın әseyd әрdures

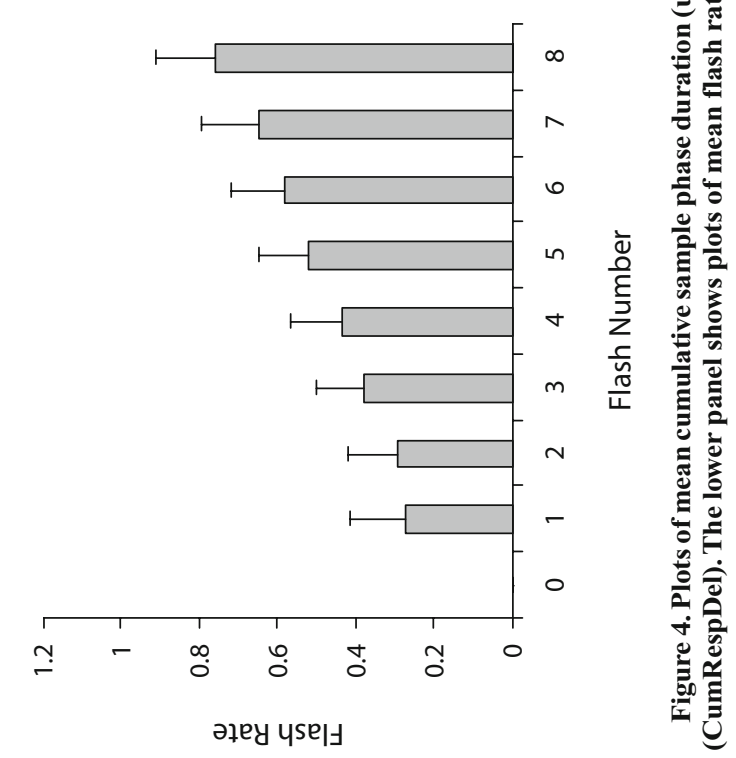


Table 3

Hierarchical Logistic Regression Results From the First 10 Sessions of Transfer Testing for All Three Conditions

\begin{tabular}{|c|c|c|c|c|c|c|c|c|}
\hline \multirow[b]{3}{*}{ Variable } & \multicolumn{8}{|c|}{ Subject } \\
\hline & \multicolumn{2}{|c|}{181} & \multicolumn{2}{|c|}{182} & \multicolumn{2}{|c|}{183} & \multicolumn{2}{|c|}{184} \\
\hline & $\mathrm{B}$ & $\operatorname{Exp}(B)$ & $\mathrm{B}$ & $\operatorname{Exp}(B)$ & $\mathrm{B}$ & $\operatorname{Exp}(B)$ & $\mathrm{B}$ & $\operatorname{Exp}(B)$ \\
\hline \multicolumn{9}{|c|}{2 vs. 6} \\
\hline Sample duration & $-0.09^{*}$ & 0.92 & $-0.09^{* *}$ & 0.91 & -0.02 & 0.98 & -0.003 & 1.00 \\
\hline Flash rate & $-2.06^{+++}$ & 0.13 & -0.8 & 0.45 & -0.89 & 0.41 & -0.68 & 0.51 \\
\hline Flash number & $1.19^{* * *}$ & 3.29 & $1.10^{* * *}$ & 3.00 & $0.98^{* * *}$ & 2.67 & $0.70^{* * *}$ & 2.01 \\
\hline Nagelkerke $R^{2}$ & \multicolumn{2}{|c|}{0.64} & \multicolumn{2}{|c|}{0.65} & \multicolumn{2}{|c|}{0.60} & \multicolumn{2}{|c|}{0.42} \\
\hline-2 log likelihood change & \multicolumn{2}{|c|}{$138.19^{* * *}$} & \multicolumn{2}{|c|}{$210.70^{* * *}$} & \multicolumn{2}{|c|}{$173.24^{* * *}$} & \multicolumn{2}{|c|}{$135.60^{* * *}$} \\
\hline \multicolumn{9}{|c|}{4 vs. 12} \\
\hline Sample duration & -0.01 & 0.99 & 0 & 1.00 & $0.07^{*}$ & 1.08 & -0.002 & 1.00 \\
\hline Flash rate & 1.35 & 3.84 & 0.27 & 1.31 & $5.33^{* * *}$ & 206.95 & 0.49 & 1.64 \\
\hline Flash number & $0.46^{* * *}$ & 1.59 & $0.53^{* * *}$ & 1.69 & $0.22^{* *}$ & 1.24 & $0.27^{* * *}$ & 1.32 \\
\hline Nagelkerke $R^{2}$ & \multicolumn{2}{|c|}{0.65} & \multicolumn{2}{|c|}{0.68} & \multicolumn{2}{|c|}{0.62} & \multicolumn{2}{|c|}{0.37} \\
\hline$-2 \log$ likelihood change & \multicolumn{2}{|c|}{$35.48^{* * *}$} & \multicolumn{2}{|c|}{$42.99^{* * *}$} & \multicolumn{2}{|c|}{$11.86^{* *}$} & \multicolumn{2}{|c|}{$29.28^{* * *}$} \\
\hline \multicolumn{9}{|c|}{8 vs. 24} \\
\hline Sample duration & -0.04 & 0.96 & -0.05 & 0.96 & -0.06 & 0.94 & $0.05^{* *}$ & 1.05 \\
\hline Flash rate & 1.71 & 5.54 & -0.23 & 0.79 & 0.82 & 2.26 & $3.10^{* * *}$ & 22.12 \\
\hline Flash number & $0.228^{* * *}$ & 1.255 & $0.380^{* * *}$ & 1.463 & $0.241^{* * *}$ & 1.272 & $0.052^{++}$ & 1.054 \\
\hline Nagelkerke $R^{2}$ & \multirow{2}{*}{\multicolumn{2}{|c|}{$\begin{array}{c}0.70 \\
18.90^{* * *}\end{array}$}} & \multirow{2}{*}{\multicolumn{2}{|c|}{$\begin{array}{c}0.80 \\
102.95^{* * *}\end{array}$}} & \multirow{2}{*}{\multicolumn{2}{|c|}{$\begin{array}{c}0.62 \\
55.38^{* * *}\end{array}$}} & \multirow{2}{*}{\multicolumn{2}{|c|}{$\begin{array}{l}0.44 \\
3.06^{+}\end{array}$}} \\
\hline$-2 \log$ likelihood change & & & & & & & & \\
\hline
\end{tabular}

\section{DISCUSSION}

Results showed that all of the pigeons were able to successfully perform the numerosity discrimination with 2-versus-6, 4-versus-12, and 8-versus-24 flashes. Although there was some covariation between temporal variables and flash number due to the response-dependent nature of the procedure, the hierarchical logistic regressions confirmed that choice responding was controlled primarily by number, rather than by sample phase duration or flash rate. This shows that pigeons are able to discriminate on the basis of relative numerosity, even in the presence of covarying temporal cues. Performance was dependent on numerical magnitude: DLs increased significantly as number increased, suggesting that the difference required for two numbers to be distinguished increased with magnitude, consistent with scalar variability and Weber's law.

This experiment extends research conducted by Emmerton and Renner (2006), demonstrating transfer of relative numerosity judgments with sequential visual stimuli up to two units outside the baseline training, as well as within the baseline training range. Weaker transfer to lower values outside the training ranges was found relative to the higher values: Although some subjects produced fewer large responses on the 0 -flash trials than on the next-tolowest flash number values, no significant differences were found between the proportion of large responses to the lowest flash numbers. This is most likely due to floor effects, since responding on the second lowest numerical trial type was often equal to or very close to 0 , restricting any further decrease. Thus, on the basis of these results alone, it is not possible to say whether the subjects were able to extrapolate their numerical understanding to lower values outside the training range.

\section{Representation and Response Rules}

BPs were closer to the arithmetic than to the geometric mean for all three conditions. This finding contrasts with previous research on numerical bisection with nonhumans, which typically has obtained BPs at the geometric mean (e.g., Fetterman, 1993; Jordan \& Brannon, 2006b; Meck \& Church, 1983; Roberts, 2005).

The only other study to obtain strong evidence for bisection at the arithmetic mean is Droit-Volet et al. (2003), which found this in both verbal and nonverbal counting of children and adults. Note that BPs' being at the arithmetic mean is predicted for verbal counting, given that human verbal representations of number are linear with constant, not scalar, variability. Jordan and Brannon (2006a) suggested that certain characteristics of their experiment might have been responsible for the anomalous BP locations in the nonverbal condition of Droit-Volet et al. Because their task involved the sequential presentation of stimuli, the items to be counted could have been enumerated by a parallel process and consequently elicited a more linear format of numerical representation. However, several bisection experiments have used sequential stimuli and still obtained BPs at the geometric mean (e.g., Fetterman, 1993; Meck \& Church, 1983; Roberts, 2005; Roberts \& Mitchell, 1994). Thus, sequential stimulus presentation does not appear to be solely responsible for the arithmetic BPs obtained in Droit-Volet et al. or in the present study.

Jordan and Brannon (2006a) also noted that subjects might have been counting subvocally in Droit-Volet et al.'s (2003) nonverbal condition due to the long duration of stimulus presentation, despite the verbal distractor task. If subjects had been counting stimuli in the same manner as in the verbal condition, BPs at the arithmetic mean should 
not be surprising. However, this does not explain the BPs obtained in this experiment, because pigeons could not have been verbally counting.

Fetterman (1993) suggested that the arithmetic mean might be a better predictor of BPs than would the geometric means for small numerical fixed ratio values (e.g., less than 15); however, the obtained difference between the arithmetic and geometric means with these values is small. The obtained BPs at the arithmetic mean for the 2 -versus- 6 and 4-versus- 12 conditions would be consistent with Fetterman's findings; however, BPs for the 8 -versus-24 condition were also located closer to the arithmetic than to the geometric mean, suggesting that this result is not limited to small numerical magnitudes only.

The location of BPs in temporal discriminations depends on several procedural factors, and these might also affect numerical bisection (Brown, McCormack, Smith, \& Stewart, 2005). One factor is whether the spacing of stimulus values is logarithmic or linear. BPs can be shifted leftward, toward the geometric mean, if items are logarithmically, rather than linearly, spaced (Allan, 2002; Wearden \& Ferrara, 1995). This effect appears to depend on the ratio of the longest/largest to shortest/smallest stimuli: With large long:short ratios (e.g., 9:1), the effect of stimulus distribution is much greater than with small long:short ratios (e.g., 4:1) (Brown et al., 2005; Wearden \& Ferrara, 1996). For humans, a long:short ratio of $2: 1$ or less will generate bisection at the geometric mean and no effect of stimulus distributions, whereas values greater than 2:1 will produce bisection at the arithmetic mean and effects of stimulus spacing. Animals, on the other hand, can still produce BPs at the geometric mean with values of up to 4:1 (Wearden \& Ferrara, 1996).

It is possible that the results of the present experiment could be explained by our use of arithmetic stimulus spacing, which may have skewed BPs toward the arithmetic mean. However, stimulus spacing effects with nonhuman animals have been found in only one temporal bisection study with rats (Raslear, 1985), which involved very large ratios of 100:1. With our greatest large:small ratio of $3: 1$, which would be considered a small ratio in terms of effects on BPs, it seems unlikely that stimulus spacing would be the primary reason for BPs' being located at the arithmetic mean.

To the extent that such procedural factors can be ruled out, the most likely explanation for our results is that the BP locations reflect the structure of the numerical representation that subjects developed. Recall that bisection at the geometric mean is generally taken as evidence for either a logarithmic scale with constant generalization between values or a linear scale with increasing generalization between values. These are the two major possibilities that have been suggested for nonverbal numerical representation, and both predict scalar variability. On the other hand, bisection at the arithmetic mean would suggest that discriminations were based on a linear representation of number, with constant variability between numerical values. This number scale is normally associated with human verbal representation of number, where values are equally spaced along the continuum and generalization does not increase with magnitude. Thus, bisection at the arithmetic mean may suggest that pigeons developed a linear representation of number with constant generalization.

However, one problem with this suggestion is that a linear representation with constant generalization does not predict scalar variability. Indeed, we found that variability was scalar: Psychometric functions from the different conditions superimposed when plotted on a relative scale, and a positive linear relationship between the BPs and the DLs was found. Relative variability also remained constant as numerical magnitude increased: The slope of Weber fractions (DL/BP) did not differ significantly from zero when plotted against BPs on a log-log scale. Thus, the nature of the representation developed by pigeons in our procedure remains unclear.

What kind of response rule did subjects use to perform this discrimination? One possibility is a proximity or likelihood ratio rule, where responses are based on the assessment of which anchor value most likely generated the test signal (Gibbon, 1981). Subjects learn the absolute values of training stimuli, and generalization around the two anchor values produces the typical bisection functions in transfer tests. This would predict bisection at the arithmetic mean if constant variance is assumed and would also predict transfer to values outside the training range, as long as the numerical distances between the sample value to each of the anchor values were not equal. Responding based on a similarity rule is determined by the relative similarity between the test value and two anchor values, based on their ratios. This rule, however, does not predict bisection at the arithmetic mean (Gibbon, 1981).

Are there other response rules that might account for the present data? It is unlikely that subjects performed the numerical bisection task by calculating the arithmetic mean as a threshold value and assessing the relative difference between the test value and the arithmetic mean, responding "small" if it was lower and "large" if it was higher. This response rule would predict response-phase choice latencies that decrease as the difference between the test values and arithmetic mean increase and judgments become easier. Our data did not support this prediction; if anything, choice latencies (not reported here) increased linearly as numerical magnitude increased.

Alternatively, responding may have been determined by comparing test values with just one of the anchor values (e.g., if X, respond "small"; if not X, respond "large"). If this were the case, it would be likely that subjects would have used the smaller anchor value as the comparator, since it would be easier and quicker to determine whether the number of flashes was equal to, say, 2 , rather than waiting for 6 . This could possibly account for the pattern seen in the choice latencies, but it is unclear what implications this would have for the location of the BP.

Although the implications of the BP and variability results for the representation of number and response rule used by pigeons may be unclear, our results show that pigeons are able to discriminate and bisect different ranges of numerical values and that responding was controlled by number above and beyond temporal variables. As stimuli were presented successively, subjects were required to 
monitor and remember the number of flashes seen during the sample phase, before being able to make their choice response. Because only one sample was presented at a time, a representation of the two anchor values, at least, must have been developed for subjects to compare test values against and to allow correct responding. Furthermore, the finding that subjects were able to extrapolate to novel numbers both within and outside the training range suggests that an understanding of relative numerosity had been developed. Further research is required to determine the nature of the underlying numerical scale used in these discriminations.

\section{AUTHOR NOTE}

This research was funded by a TEC Bright Futures Top Achiever Doctoral Scholarship and was conducted at the University of Canterbury, New Zealand, as part of the doctoral research of the first author. L.T. is now at Reed College, Portland, OR. Correspondence concerning this article and reprint requests should be addressed to L. Tan or to R. C. Grace, Department of Psychology, University of Canterbury, Christchurch, New Zealand (e-mail: 1tan@reed.edu; randolph.grace@canterbury.ac.nz).

\section{REFERENCES}

Allan, L. G. (2002). The location and interpretation of the bisection point. Quarterly Journal of Experimental Psychology, 55B, 43-60.

Alsop, B., \& HonIG, W. K. (1991). Sequential stimuli and relative numerosity discriminations in pigeons. Journal of Experimental Psychology: Animal Behavior Processes, 17, 386-395.

Beran, M. J. (2001). Summation and numerousness judgments of sequentially presented sets of items by chimpanzees (Pan troglodytes). Journal of Comparative Psychology, 115, 181-191.

Beran, M. J. (2004). Chimpanzees (Pan troglodytes) respond to nonvisible sets after one-by-one addition and removal of items. Journal of Comparative Psychology, 118, 25-36.

Beran, M. J. (2007). Rhesus monkeys (Macaca mulatta) enumerate large and small sequentially presented sets of items using analog numerical representations. Journal of Experimental Psychology: Animal Behavior Processes, 33, 42-54.

BRANNON, E. M. (2006). The representation of numerical magnitude. Current Opinion in Neurobiology, 16, 222-229.

Brown, G. D. A., McCormack, T., Smith, M., \& Stewart, N. (2005). Identification and bisection of temporal durations and tone frequencies: Common models for temporal and nontemporal stimuli. Journal of Experimental Psychology: Human Perception \& Performance, 31, 919-938.

Christensen, D. R., \& Grace, R. C. (2008). Rapid acquisition in concurrent chains: Effects of initial-link duration. Behavioural Processes, 78, 217-223.

Cordes, S., Gelman, R., Gallistel, C. R., \& Whalen, J. (2001). Variability signatures distinguish verbal from nonverbal counting for both large and small numbers. Psychonomic Bulletin \& Review, 8, 698-707.

Davis, H., \& Pérusse, R. (1988). Numerical competence in animals: Definitional issues, current evidence, and a new research agenda. $B e-$ havioral \& Brain Sciences, 11, 561-579.

Droit-Volet, S., Clément, A., \& Fayol, M. (2003). Time and number discrimination in a bisection task with a sequence of stimuli: A developmental approach. Journal of Experimental Child Psychology, 84, 63-76.

EMmerton, J. (1998). Numerosity differences and effects of stimulus density on pigeons' discrimination performance. Animal Learning \& Behavior, 26, 243-256.

EMmerton, J., \& RenNer, J. C. (2006). Scalar effects in the discrimination of numerosity by pigeons. Learning \& Behavior, 34, 176-192.

Fernandes, D. M., \& Church, R. M. (1982). Discrimination of the number of sequential events by rats. Animal Learning \& Behavior, 10, 171-176.

Fetterman, J. G. (1993). Numerosity discrimination: Both time and number matter. Journal of Experimental Psychology: Animal Behavior Processes, 19, 149-164.

Fetterman, J. G., Dreyfus, L. R., \& Stubbs, D. A. (1985). Scaling of response-based events. Journal of Experimental Psychology: Animal Behavior Processes, 11, 388-404.

Fetterman, J. G., Stubbs, D. A., \& Dreyfus, L. R. (1986). Scaling of events spaced in time. Behavioural Processes, 13, 53-68.

Fleshler, M., \& Hoffman, H. S. (1962). A progression for generating variable-interval schedules. Journal of the Experimental Analysis of Behavior, 5, 529-530.

GibBon, J. (1981). On the form and location of the psychometric bisection function for time. Journal of Mathematical Psychology, 24, 58-87.

Hanus, D., \& Call, J. (2007). Discrete quantity judgments in the great apes (Pan paniscus, Pan troglodytes, Gorilla gorilla, Pongo pygmaeus): The effect of presenting whole sets versus item-by-item. Journal of Comparative Psychology, 121, 241-249.

Hauser, M. D., Carey, S., \& Hauser, L. B. (2000). Spontaneous number representation in semi-free-ranging rhesus monkeys. Proceedings of the Royal Society B, 267, 829-833.

Honig, W. K., \& Stewart, K. E. (1989). Discrimination of relative numerosity by pigeons. Animal Learning \& Behavior, 17, 134-146.

JoRdAN, K. E., \& BranNON, E. M. (2006a). A common representational system governed by Weber's law: Nonverbal numerical similarity judgments in 6-year-olds and rhesus macaques. Journal of Experimental Child Psychology, 95, 215-229.

Jordan, K. E., \& BRANnON, E. M. (2006b). Weber's law influences numerical representations in rhesus macaques (Macaca mulatta). Animal Cognition, 9, 159-172.

MecK, W. H., \& ChURCh, R. M. (1983). A mode control model of counting and timing processes. Journal of Experimental Psychology: Animal Behavior Processes, 9, 320-334.

Olthof, A., IDEN, C. M., \& RoberTs, W. A. (1997). Judgments of ordinality and summation of number symbols by squirrel monkeys (Saimiri sciureus). Journal of Experimental Psychology: Animal Behavior Processes, 23, 325-339.

Raslear, T. G. (1985). A test of the Pfanzagl bisection model in rats. Journal of Experimental Psychology: Animal Behavior Processes, 9, 49-62.

Rilling, M., \& McDiarmid, C. (1965). Signal detection in fixed-ratio schedules. Science, $148,526-527$.

RoBERTS, W. A. (2005). How do pigeons represent numbers? Studies of number scale bisection. Behavioural Processes, 69, 33-43.

Roberts, W. A., \& Mitchell, S. (1994). Can a pigeon simultaneously process temporal and numerical information? Journal of Experimental Psychology: Animal Behavior Processes, 20, 66-78.

Rumbaugh, D. M., Savage-Rumbaugh, S., \& Hegel, M. T. (1987). Summation in the chimpanzee (Pan troglodytes). Journal of Experimental Psychology: Animal Behavior Processes, 13, 107-115.

Tan, L., Grace, R. C., Holland, S., \& Mclean, A. P. (2007). Numerical reproduction in pigeons. Journal of Experimental Psychology: Animal Behavior Processes, 33, 409-427.

vanMarle, K., Aw, J., McCrinK, K., \& Santos, L. R. (2006). How capuchin monkeys (Cebus apella) quantify objects and substances. Journal of Comparative Psychology, 120, 416-426.

Wearden, J. H., \& Ferrara, A. (1995). Stimulus spacing effects in temporal bisection by humans. Quarterly Journal of Experimental Psychology, 48B, 289-310.

Wearden, J. H., \& Ferrara, A. (1996). Stimulus range effects in temporal bisection by humans. Quarterly Journal of Experimental Psychology, 49B, 24-44.

(Manuscript received February 20, 2010; revision accepted for publication April 30, 2010.) 